\title{
Man Feeling Tired and "Lousy" Five Days FOLLOWING MYOCARDIAL INFARCTION
}

Andra Popescu, MD

\section{Case Presentation}

A 70 year-old male presents to his cardiologist's office with complaints of feeling fatigued and "lousy". His past medical history is significant for HTN and HL, but until a recent hospitalization, he was non-compliant with his outpatient medical regimen. Of note, he also has a history of heavy alcohol consumption and a 30 pack-year smoking history. The patient reports that he was recently discharged after acute myocardial infarction (AMI) from an outside hospital. At that time, he underwent a diagnostic cardiac catheterization, but no intervention was performed secondary to 100\% RCA stenosis and technical difficulties. He was sent home on clopidogrel, aspirin, metoprolol, lisinopril and simvastatin. He currently appears tired and anxious, however he has no symptoms of chest pain, dyspnea, orthopnea, diaphoresis, or lightheadedness; he does report that his primary symptoms leading him to admission was chest pain and shortness of breath.

On physical exam, the patient's blood pressure is $75 / 56 \mathrm{mmHg}$ with a heart rate of 89 beats per minute, respiratory rate is 18 , and his room air oxygen saturation is $98 \%$. Cardiac exam revealed a new apical systolic murmur best heard at the posterior axillary line radiating towards to the scapula with an $\mathrm{S} 3$ present. JVP was estimated to be $20 \mathrm{~cm} \mathrm{H}_{2} \mathrm{O}$. Lung exam demonstrated bibasilar crackles. Peripheral extremities were warm with no lower extremity edema.

Initial laboratory data include a creatinine of $2.7 \mathrm{mg} / \mathrm{dL}$ (baseline $1.3 \mathrm{mg} / \mathrm{dL}$ ), hemoglobin of $11.8 \mathrm{~g} / \mathrm{dL}$, a white blood cell count of 12,500 cells $/ \mathrm{mm} 3$ without left shift or band forms, and troponin of $5.89 \mathrm{ng} / \mathrm{mL}$. EKG revealed a normal sinus rhythm with ST elevations in leads II, III, aVF and ST depressions throughout the lateral precordial leads suggesting ongoing inferolateral ischemia or injury.

The patient was admitted to the Cardiac Care Unit (CCU) for further medical management. He received fluid boluses for BP support. Aspirin and statin therapy were continued and additionally, therapeutic intravenous heparin was initiated. Clopidogrel was held due to a potential need for surgical intervention. Beta blocker and ACE inhibitor were discontinued secondary to hypotension.

Cardiac catheterization reports were obtained from the outside hospital and demonstrated a 100\% RCA stenosis. Additionally, there was diffuse disease throughout the coronary arteries including a $50 \% \mathrm{LAD}, 50 \% \mathrm{LCx}, 50 \% \mathrm{OM} 1$, and $60 \% \mathrm{D} 2$ stenosis with an ejection fraction of $35 \%$. An echocardiogram obtained upon admission to this institution visualized a mobile echodensity attached to the chordal region of the anterior mitral leaflet suggestive of partial papillary muscle rupture leading to anterior leaflet prolapse, as well as systolic dysfunction due to multiple segmental wall motion abnormalities. Doppler evaluation confirmed severe, posteriorlaterally directed mitral regurgitation with a "shoulder" sign, suggestive of severe, acute MR likely secondary to papillary muscle rupture.

Due to the patient's acute renal failure, RCA angioplasty was not performed despite persistent ST elevations suggestive of ongoing ischemia. Right heart catheterization was performed which demonstrated a RA pressure of $10 \mathrm{mmHg}, \mathrm{RV}$ pressure of 43/15 $\mathrm{mmHg}$, PA pressure of $42 / 23 \mathrm{mmHg}$, and a pulmonary capillary wedge pressure of $21 \mathrm{mmHg}$ without significant $\mathrm{v}$-wave. Cardiac index was measured at $1.5 \mathrm{~L} / \mathrm{min} / \mathrm{m} 2$, consistent with cardiogenic shock. Intra-aortic balloon counterpulsation was not performed due to the presence of severe PVD. The patient was started on a dopamine drip and taken to the operating room for emergent coronary bypass revascularization and mitral valve replacement.

The post-operative course was complicated by cardiac arrest and ventricular fibrillation. A trans-esophageal echocardiogram following resuscitation revealed a new $2 \mathrm{~mm}$ inferoseptal VSD. Multi-system organ failure ensued and the patient expired 9 days following bypass surgery.

\section{Discussion}

This is an excellent example for a discussion of mechanical complications following myocardial infarction. Our patient had two of the three major complications: rupture of the free wall, rupture of the ventricular septum and mitral regurgitation, each of which can result in cardiogenic shock.

1. Rupture of the ventricular free wall is a very serious and often lethal complication which usually occurs within the first 5 days post MI and has an incidence of less than 1\% when all AMI patients are considered, ${ }^{1}$ but its incidence is between 14-26\% among patients dying of acute MI. ${ }^{2,3}$ Risk factors for free wall rupture were large ischemia territory, lack of collateral blood flow (no history of previous angina or MI), ${ }^{3}$ anterior MI, age> 70 , and female sex. ${ }^{4.5}$ Rupture typically occurs in an area that has been infarcted without successful reperfusion. ${ }^{6}$ There was an increased occurrence noted in patients treated with thrombolytic therapy versus patients who underwent coronary intervention (3.3\% vs. $1.8 \%) .{ }^{5}$ Complete rupture of the left ventricular free wall is suggested by the development of sudden right heart failure and shock due to hemopericardium with resultant electromechanical dissociation. Incomplete, or subacute, rupture occurs when organized thrombus and pericardium seal the perforation forming a pseudoaneurysm. Management is with fluids resuscitation, ionotropic and/or vasopressor support, pericardiocentesis, IABP counterpulsation, and percutaneous cardiopulmonary bypass. Surgical repair is indicated for pseudoaneurysm. Prognosis is very poor and mortality is exceptionally high. 
2. Rupture of the interventricular septum typically occurs 3-5 days after an acute MI, however, it may develop within the first 24 hours or as late as two weeks. Risk factors include "wrap around" LAD covering a large myocardial territory, ${ }^{10,11}$ extensive myocardial damage, poor septal collateral circulation, or RV infarction. ${ }^{7.89}$ Incidence has decreased from $2 \%$ in the conventional therapy era to $0.2 \%$ in the reperfusion therapy era. ${ }^{12,13}$ Although not a predisposing factor for septal rupture, thrombolytics may precipitate the occurrence. Rupture develops at the margin of the necrotic and non-necrotic myocardium, typically in the apical septum with anterior MI or near the base of in inferior MI. The size of the defect determines the magnitude of the left to right shunt, which will affect the survival. Presentation is acute hemodynamic compromise with hypotension, biventricular failure and a new, loud, harsh, holosystolic murmur best heard at the lower left and right sternal borders. ECG may show persistent ST elevations for more the 72 hours in those do not undergo reperfusion. Defects can be visualized by echocardiogram and confirmed with Swan-Ganz catheterization via the measurement of a "step-up" in the oxygen saturation right atrial and ventricular chambers. Patients with HF and shock require emergent surgical repair, while delayed surgical management is feasible in patients with HF without shock. Transcatheter closure of a post-infarction VSD is another potential approach which may be appropriate for patients who otherwise are not candidates for other surgical treatments.

3. Acute mitral regurgitation following myocardial infarction is due to ischemic papillary muscle dysfunction, LV dilatation or true aneurysm, or papillary muscle/chordal rupture. The degree of MR may vary from mild (which may be transient during ischemia) to moderate-severe MR (which may be due to papillary muscle or chordal rupture). ${ }^{14}$ Papillary muscle rupture is seen both in STEMI and NSTEMI, ${ }^{12,14}$ and accounts for 5\% of deaths seen in AMI. It is usually seen 2-7 days post-MI and more frequently affects the posteromedial papillary due to its sole blood supply from the PDA, whereas the anterolateral papillary muscle has a dual blood supply from LAD and LCx. Acute MR occurs more frequently in patients presenting with their first infarction without established collateralization and up to $50 \%$ of these patients may have only single vessel disease. Presentation is acute onset of hypotension and pulmonary edema with a hyperactive precordium and a mid-, late- or holosystolic murmur that has widespread radiation. Intensity of the murmur does not necessarily correlate with the severity of the regurgitation. As many as $50 \%$ of patients who have severe MR due to rupture have early equalization of left ventricular and left atrial pressures resulting in silent $M R$ or a short, soft, indistinct murmur. Clinical diagnosis is confirmed by echocardiogram, which may demonstrate a flail segment of the mitral valve, a severed papillary muscle, or chordae moving freely within the left ventricular cavity. Swan-Ganz catheterization usually reveals giant $\mathrm{v}$-waves. Medical treatment includes aggressive reduction of afterload using nitrates, sodium nitroprusside, diuretics and IABP counterpulsation. Emergent surgery remains the treatment of choice for papillary muscle rupture, although operative mortality is high $(20-25 \%){ }^{15}$ Mitral valve repair is superior to replacement and should be attempted in experienced centers when there is no papillary necrosis present..$^{14,16}$

\section{References}

1. Patel, MR, Meine, TJ, Lindblad, L, et al. Cardiac tamponade in the fibrinolytic era: analysis of $>100,000$ patients with ST-segment elevation myocardial infarction. Am Heart J 2006; 151:316.

2. Stevenson, WG, Linssen, GC, Havenith, MG, et al. The spectrum of death after myocardial infarction: A necropsy study. Am Heart J 1989; 118:1182

3. Pohjola-Sintonen, S, Muller, JE, Stone, PH, et al. Ventricular septal and free wall rupture complicating acute myocardial infarction: experience in the Multicenter Investigation of Limitation of Infarct Size. Am Heart J 1989; 117:809

4. Becker, RC, Hochman, JS, Cannon, CP, et al, for the TIMI 9 Investigators. Fatal cardiac rupture among patients treated with thrombolytic agents and adjunctive thrombin antagonists. Observations from the Thrombolysis and Thrombin Inhibition in Myocardial Infarction 9 study. J Am Coll Cardiol 1999; 33:479.

5. Moreno, R, Lopez-Sendon, J, Garcia, E, et al. Primary angioplasty reduces the risk of left ventricular free wall rupture compared with thrombolysis in patients with acute myocardial infarction. J Am Coll Cardiol 2002; 39:598

6. Cheriex, EC, de Swart, H, Dijkman, LW, et al. Myocardial rupture after myocardial infarction is related to the perfusion status of the infarct-related coronary artery. Am Heart J 1995; 129:644

7. Radford, MJ, Johnson, RA, Daggett, EM Jr, et al. Ventricular septal rupture: A review of clinical and physiologic features and an analysis of survival. Circulation $1981 ; 64: 545$

8. Skehan, JD, Carey, C, Norrell, MS, et al. Patterns of coronary artery disease in postinfarction ventricular septal rupture. Br Heart J 1989; 62:268

9. Pretre, R, Rickli, H, Ye, Q, et al. Frequency of collateral blood flow in the infarctrelated coronary artery in rupture of the ventricular septum after acute myocardial infarction. Am J Cardiol 2000; 85:497.

10. Hayashi, T, Hirano, Y, Takai, H, et al. Usefulness of ST-segment elevation in the inferior leads in predicting ventricular septal rupture in patients with anterior wall acute myocardial infarction. Am J Cardiol 2005; 96:1037

11. Sasaki, K, Yotsukura, M, Sakata, K, et al. Relation of ST-segment changes in inferior leads during anterior wall acute myocardial infarction to length and occlusion site of the left anterior descending coronary artery. Am J Cardiol 2001; 87:1340.

12. Reeder, GS. Identification and treatment of complications of myocardial infarction. Mayo Clin Proc 1995; 70:880

13. Pierli, C, Lisi, G, Mezzacapo, B. Subacute left ventricular free wall rupture. Surgical repair prompted by echocardiographic diagnosis. Chest 1991; 100:1174.

14. Lavie, CJ, Gersh, BJ. Mechanical and electrical complications of acute myocardial infarction. Mayo Clin Proc 1990; 65:709.

15. Pasternak, RC, Braunwald, E, Sobel, BE. Acute myocardial infarction. In: Heart Disease, 4th ed, Braunwald, EB (Ed), Saunders, Philadelphia 1992. p.200.

16. David, TE. Techniques and results of mitral valve repair for ischemic mitral regurgitation. J Card Surg 1994; 9:274. 